\title{
RECHTSERNEUERUNG UND ISLAM IN SOMALIA
}

\author{
KonRAD Dilger
}

\section{Allgemeines}

Am 1. 7. 1960 entstand Somalia als selbständiger Staat, als das britische Protektorat Somaliland $^{1}$ im Norden und das unter italienischer Verwaltung stehende Treuhandgebiet ${ }^{2}$ am südlichen Küstenstreifen unabhängig wurden und sich zu einem einheitlichen Staat zusammenschlossen. Die „Republik Somalia“, wie ihr Name zunächst lautete, heißt seit dem Staatsstreich vom 21. 10. 1969 „,Demokratische Republik Somalia“ und gehört der Arabischen Liga an. Somalis leben außerdem in dem ehemals französischen Territorium der Afar und Issas mit der Hauptstadt Djibouti, das erst im Frühsommer des Jahres 1977 unabhängig geworden ist, in der zu Äthiopien gehörenden und umstrittenen Provinz Ogaden sowie im nördlichen Grenzbereich von Kenia. Der größte Teil der knapp 3 Millionen Einwohner von Somalia ${ }^{3}$ sind Muslime, die innerhalb des sunnitischen Islam der schafiitischen Richtung angehören" ${ }^{4}$.

Der mit der Erlangung der Unabhängigkeit erwachte Nationalismus führte in Somalia zu einer starken Betonung des Islam ${ }^{5}$. Das zeigt sich bereits in der Verfassung vom 21. 6. 1960, die an sich grundsätzlich nach italienischem Vorbild gestaltet worden ist ${ }^{6}$. So ist gemäß Art. 1 Abs. 3 der Verfassung der Islam Staatsreligion. Nach Art. 29 der Verfassung ist es daher nicht erlaubt, irgendeine andere Religion als den Islam zu verbreiten ${ }^{7}$. Laut Art. 35 der Verfassung ist islamischer Religionsunterricht für alle Schüler, die dem Islam angehören, vorgeschrieben. Der Präsident der Republik muß nach Art. 71 Abs. 1 der Verfassung ein Muslim sein. Außenpolitisch hat sich die Somalische Republik zum Programm gemacht, die Solidarität zwischen den Völkern der Welt, insbesondere den afrikanischen und islamischen Völkern zu ermutigen (Art. 6 Abs. 4 der Verfassung) ${ }^{8}$. Die Betonung der gemeinsamen islamischen Tradition in den beiden Landesteilen stellt das geeignete Mittel dar, das wichtigste politische Ziel des jungen Staatswesens zu verwirklichen, nämlich die Integration der ehemals unter italienischer und britischer Verwaltung stehenden beiden Landesteile zu einem Staatsganzen. Um die erforderliche Rechtseinheit zu verwirklichen, ist gerade auch im rechtlichen Bereich die islamische Komponente herausgestellt worden.

1 Zwischen Britisch-Somaliland und Großbritannien bestanden seit 1894 Schutz verträge. Am 26. 6. 1960 wurde das britische Protektoratsgebiet unabhängig.

2 Das Protektorat über das von Italien beherrschte Gebiet war 1899 errichtet worden. Seit 1936 war es Teil des italienischen OstafrikaBesitzes, im Jahre 1950 wurde es UN-Treuhandgebiet.

3 So die Angabe für 1972.

4 Danebengibt es auchnoch zaiditische Araber, die zu denSchiiten zählen. Vgl. Contini, TheSomali Republic: An Experiment in Legal Integration (1969) VII und Lexikon der islamischen Welt, hrsg. von Kreiser/Diem/Majer (1974) III s. v. Somalia; sowie über die Zaiditen ebenda III s. v. Zaiditen; besonders aber auch Strothmann, Kultus der Zaiditen (1912); ders., al-Zaidiya: Enzyklopädie des Islam IV (1934) 1295.

5 Zur Rolle des Islam in Hinblick auf die politische Entwicklung im Nahen Osten vgl. Dilger, Die Stärkung des islamischen Rechts in Afrika als Folge der Emanzipation afrikanischer Mitgliedsstaaten der Arabischen Liga: Die Welt des Islam 18 (1978) $153-177$.

6 Die Verfassung von Somalia findet sich bei Blaustein/Flanz, Constitutions of the Countries of the World, s. v. Somali Democratic Republic (September 1971, bearb. von Ganzglass).

7 Verfassungsänderung durch Gesetz Nr. 16/1963 vom 29. 6. 1963. Vgl. den ursprünglichen Wortlaut bei Contini (s. Anm. 4) 58 Anm. 218.

8 Der Begriff der Solidarität bedeutet eine Anspielung auf den arabischen Sozialismus, dessen zentrales Element die soziale Solidarität (tadamun igtima'i) mit gegenseitiger Verantwortlichkeit(takaful) ist. Vgl. zu dem BegriffDilger, Eigentumsordnung und Sozialismus in den arabischen Staaten: Zeitschrift für vergleichende Rechtswissenschaft 75 (1976) 171-201 (179 f.).

9 Art. 50 der Verfassung lautet (in englischer Ubersetzung): The doctrine of Islam shall be the main source of the laws of this state. 
So bildet gemäß Art. 50 der Verfassung der Islam die Hauptquelle des Rechts ${ }^{9}$. Alle Gesetze und gesetzlichen Vorschriften müssen nicht nur mit der Verfassung übereinstimmen, sondern auch mit den allgemeinen Prinzipien des Islam (Art. 98 Abs. 1 der Verfassung). Damit sind bereits in der Verfassung Grundsätze ausgesprochen, die für die gesamte Rechtsentwicklung von größter Bedeutung geworden sind. Zwar ist die Verfassung knapp ein Jahrzehnt nach Staatsgründung im Anschluß an den Staatsstreich vom 21. 10. 1969 durch das Dekret Nr. 38 vom 24. 2. 1970 suspendiert worden, nachdem schon durch die erste Charta am Tag des Staatsstreichs alle Bestimmungen der Verfassung aufgehoben worden waren, die dem Geist der Revolution widersprachen ${ }^{10}$. Aber die den Islam und die Stellung des islamischen Rechts in Somalia betreffenden Grundsätze sind über diesen Zeitpunkt hinaus bis heute bestimmend geblieben.

\section{Vereinheitlichung der Gerichtsbarkeit}

Im Zeitpunkt der Unabhängigkeit sah sich Somalia einer vierfachen Rechtstradition gegenüber: im Norden des Landes galt Recht britischer Prägung, im Süden war das Recht italienischen Ursprungs in Geltung, während daneben im ganzen Land islamisches Recht (Scheriatrecht) und Gewohnheitsrecht, das sich größtenteils aus dem Scheriatrecht entwickelt hat, in der einheimischen Bevölkerung maßgebend waren. Der Vielfalt im materiellen Recht entsprach die Gerichtsbarkeit, die nach italienischem und angelsächsischem System ausgebaut worden war. Ohne auf die Gliederung des Gerichtswesens im einzelnen einzugehen, sei nur erwähnt, daß es auch im britischen Protektorat - abweichend von Aden und Indien etwa $a^{11}$ religiöse islamische Gerichte gegeben hat ${ }^{12}$. Die Vereinheitlichung der Gerichtsbarkeit erfolgte 1962 durch das Gesetz über die ,,Organization of the Judiciary“, das durch Gesetzesdekret Nr. 3/1962 vom 12. 6. 1962 erlassen worden war ${ }^{13}$. Abgesehen von der Integration des Gerichtswesens in den ehemals getrennten Landesteilen war damals die wichtigste Frage, ob neben den ordentlichen Gerichten die islamischen Scheriatgerichte bestehen bleiben. Auf die Diskussion dieser Frage und die darin vorgebrachten Argumente soll an dieser Stelle nicht weiter eingegangen werden ${ }^{14}$. Im Endergebnis wurden auch die Scheriatgerichte in den allgemeinen Gerichtsaufbau eingefügt, so daß es heute in Somalia nur noch eine einheitliche Gerichtsbarkeit gibt.

\section{Vereinheitlichung des materiellen Rechts}

Während der Treuhandzeit ${ }^{15}$ waren die Scheriatgerichte in der italienischen Zone gemäß dem Gesetz über die Organisation der Gerichtsbarkeit von 1956 (Ordinance Nr. 5 vom 2. 2. 1956) zuständig für alle Zivilsachen zwischen Muslimen (einschließlich der Angelegenheiten

\footnotetext{
10 Vgl. dazu Noor Muhammad, The Legal System of the Somali Democratic Republic (1972) 276 und Blaustein/Flanz (s. Anm. 6) s. v. Somali Democratic Republik 6 f.

11 Zum Fehlen der Scheriatgerichte in Aden und Indien vgl. Dilger, Das Recht unter dem Einfluß des Sozialismus in der Volksrepublik Jemen: Zeitschrift für vergleichende Rechtswissenschaft 75 (1975) 1-44 (8).

12 Vgl. im einzelnen Contini $32 \mathrm{ff}$.

13 Das Gesetz über die Organization of the Judiciary (durch Gesetzesdekret Nr. 3/1962 vom 12. 6. 1962) ist in der ehemaligen britischen Nordregion ab 1. 9. 1962 wirksam geworden, in der ehemals italienischen Südregion erst ab 1. 10. 1963.

14 Vgl. zur Diskussion über das Fortbestehen der Scheriatgerichte Noor Muhammad (s. Anm. 10) 226.

15 Zur früheren Epoche vgl. Noor Muhammad (s. Anm. 10) $89 \mathrm{ff}$.
} 
des Personalstatuts) ${ }^{\mathbf{1 6}}$, außer der Kläger zog es vor, sich der Gerichtsbarkeit des regionalen Richters und damit italienisch orientiertem Recht zu unterwerfen. Außerdem war der regionale Richter immer dann ausschließlich zuständig, wenn Rechtsstreitigkeiten auf einem Schriftstück basierten, sowie in Fällen, in denen die Rechtsbeziehungen mit Förmlichkeiten in Zusammenhang standen, die nicht dem islamischen Recht oder dem Gewohnheitsrecht entsprachen (Art. 2 der Ordinance Nr. 5). Der Grund dafür war, daß das islamische Recht nur Zeugenaussagen als vollwertiges Beweismittel kennt und Urkunden nur eine untergeordnete Bedeutung zukommt. Im strafrechtlichen Bereich waren die Scheriatgerichte im italienischen Landesteil für alle Straftaten zuständig, die durch Muslime zum Schaden anderer Glaubensbrüder begangen worden waren ${ }^{\mathbf{1 7}}$. Soweit die religiösen islamischen Richter (qadi) zuständig waren, wurde naturgemäß islamisches Recht angewendet.Islamisches Recht galt also grundsätzlich in allen Rechtsfragen, die Muslime betrafen. Die Frage der Anwendung islamischen Rechts war an den betroffenen Personen orientiert. Erst im Jahre 1958 wurde die Zuständigkeit der Scheriatsgerichtsbarkeit durch eine Gesetzesnovelle (Gesetz Nr. 14 vom 27. 5. 1958) auf den Bereich des Privatrechts beschränkt. Damit entfiel die Kompetenz der Scheriatgerichte in Strafsachen.

In der britischen Region war die Zuständigkeit der Scheriatgerichte aufgrund des Art. 10 Abs. 2 der Subordinate Courts Ordinance vom 1. 7. 1944 auf die Angelegenheiten des Personalstatuts beschränkt ${ }^{18}$. Hier hatten sie von vornherein keine strafrechtlichen Funktionen. Nach Gründung der somalischen Republik mußte eine einheitliche Lösung für die Anwendung des islamischen Rechts gefunden werden. Gemäß Art. 30 Abs. 2 der Verfassung wird das Personalstatut von Muslimen nach den ,, allgemeinen Prinzipien des islamischen Scheriatsrechts" beurteilt. Alle familien- und erbrechtlichen Fragen von Muslimen unterliegen damit (auch heute noch) im ganzen Land wie in der Kolonialzeit dem islamischen Recht. Im übrigen wird in Art. 9 des Gesetzes über die Organisation der Gerichtsbarkeit bestimmt, daß islamisches Recht - unabhängig von der Religion der Beteiligten! - in den zivilrechtlichen Streitigkeiten zur Anwendung kommt, in denen der Klagegrund sich aus dem islamischen Recht ergibt ${ }^{19}$. In allen andern Fällen gelten die positiv-rechtlichen Vorschriften. Anders als früher in der italienischen Region entscheidet also nun die Rechtsnatur der Obligation über die Frage des maßgeblichen Rechts. Damit war ein Kompromiß gefunden, durch den der Anwendungsbereich des islamischen Rechts in der ehemals britischen Zone ausgedehnt wurde, während in der früheren italienischen Zone die einseitige Wahl des Rechtsweges durch den Kläger entfiel. Den Parteien - und zwar nicht nur den muslimischen - blieb es jedoch unbenommen, ihre vertraglichen Beziehungen auf der Grundlage des Zivil- oder Scheriatsrechts zu gestalten und sich damit dem einen oder anderen Recht zu unterwerfen. Von den konservativen Kreisen wurde demgegenüber die Ansicht vertreten, daß schlechthin islamisches Recht angewendet werden müßte, weil Somalia schließlich ein islamischer Staat sei.

16 Zum Begriff des Personalstatuts vgl. Makarov, Personalstatut und persönlicher Status, in: Rechtsvergleichung und Rechtsvereinheitlichung (Festschr. zum 50jährigen Bestehen des Instituts für ausländisches und internationales Privat- und Wirtschaftsrecht der Universität Heidelberg, 1967). - Zum Personalstatut im Sinn des islamischen Rechts (ahwal sahsiya) gehören vor allem das Familien- und Erbrecht, aber auch die Schenkung (als unentgeltliche Verfügung unter Lebenden), der Vorkauf (suf'a) und die religiösen Stiftungen (waqf).

17 Im einzelnen vgl. dazu Noor Muhammad (s. Anm. 10) $93 \mathrm{ff}$

18 Vgl. zur Rechtslage im britischen Protektorat Anderson, Islamic Law in Africa (1954), 40--57 (43 f.). Zum Strafrecht in Somalia vgl. insbesondere Quellen und Schrifttum des Strafrechts, hrsg. vom Max-Planck-Institut für ausländisches und internationales Strafrecht (1972 ff.), s. v. Somalia (bearb. von Bosch).

19 Der Text von Art. 9 findet sich in englischer Ubersetzung bei Noor Muhammad (s. Anm. 10) 215. 


\section{Islamisches Recht und Verfassung als Maßstab}

Unter dengleichen Voraussetzungen, die für das Scheriatrecht gelten, ist Gewohnheitsrecht im zivilrechtlichen Bereich beachtlich. Doch war dessen Anwendung von vornherein durch die Verfassung beschränkt; denn gemäß Art. 98 Abs. 1 der Verfassung müssen alle Rechtsvorschriften mit der Verfassung und vor allem mit den ,,allgemeinen Prinzipien des Islam“ (general principles of Islam) übereinstimmen. Daher mußten die Gerichte seit dem Zusammenschluß des Landes jegliches Gewohnheitsrecht an der Verfassung und dem islamischen Recht messen und es notfalls korrigieren. In einem einheitlichen Gerichtswesen unterliegt das Gewohnheitsrecht verständlicherweise einer viel schärferen Kontrolle, als dies bei seiner Anwendung durch Gerichte geschehen würde, die voneinander unabhängig sind ${ }^{20}$. Daraus ergaben sich mitunter schwerwiegende Eingriffe in das Gewohnheitsrecht, das in einem Volk mit einem hohen Anteil an Nomaden ${ }^{21}$ naturgemäß eine Rolle spielt.

Es folgen nunmehr einige Beispiele, mit denen gezeigt werden soll, in welcher Weise das islamische Recht und die Verfassung als Maßstab für das Gewohnheitsrecht herangezogen worden sind:

1. Beispiel: In einem von dem Supreme Court entschiedenen Fall war ein Mädchen namens Amina gegen ihren Willen von ihrem Bruder verlobt worden. Doch sie ging mit ihrem Geliebten durch und heiratete diesen. Der vor den Kopf gestoßene Verlobte klagte gegen den Ehemann gemäß Gewohnheitsrecht auf die Zahlung von haal, d. h. eine Art Schadensersatz wegen Ehrverletzung ${ }^{22}$. An sich wäre die Klage nach dem Gewohnheitsrecht begründet gewesen. Aber nach dem islamischen Recht hat nicht einmal der Vater das Recht, seine volljährige Tochter ohne ihre Einwilligung zu verheiraten. Auch nach dem Minhag at-Talibin ${ }^{23}$, einem Grundwerk der schafiitischen Rechtsschule, kann ein Bruder seine erwachsene Schwester, die noch Jungfrau ist, nur verheiraten, sofern sie keinen Einspruch erhebt. Daher war nach Ansicht des Supreme Court eine wirksame Verlobung gegen den Willen der Frau nicht möglich. Da jedoch eine Klage auf haal eine gültige Verlobung voraussetzt, wurde die Klage auf Schadensersatz abgewiesen. Das Gewohnheitsrecht wurde inhaltlich auf den Umfang des islamischen Rechts reduziert ${ }^{24}$.

2. Beispiel: Der folgende vom Supreme Court entschiedene Fall zeigt, welche Ưberlegungen zur Verfassungsmäßigkeit des Gewohnheitsrechts angestellt worden sind. Nachdem ein Mädchen bei einem Verkehrsunfall ums Leben gekommen war, verlangte die Familie der Getöteten die Zahlung der diya, d. h. des ,Blutgeldes“, das nach dem islamischen Recht bei Tötung oder Körperverletzung durch den Stammesverband des Verursachers zu zahlen ist ${ }^{\mathbf{2 5}}$. Da nach Art. 43 der Verfassung die strafrechtliche Verantwortlichkeit persönlicher Natur und jede kollektive Bestrafung verboten ist, war vom Gericht der rechtliche Charakter der diya zu prüfen und zu klären, ob ihr Kollektivcharakter zukomme. Obwohl der Supreme Court in seiner Entscheidung richtig davon ausging, daß der diya im islamischen Recht

\footnotetext{
20 So zu Recht Contini (s. Anm. 4) 66.

21 In Somalia sind rund zwei Drittel der Bevölkerung Nomaden; vgl. Contini (s. Anm. 4) VII.

22 Vgl. Noor Muhammad (s. Anm. 10) 216 ff.; Contini (s. Anm. 4) 66 ff. und 71 ff.; Scek Nur, Grundlagen und Grundzüge der somalischen Verfassungs- und Rechtsordnung(Diss. 1971) $33 \mathrm{ff}$.; Contini, The Evolution of Blood-money for Homicide in Somalia: Journal of African Law 1971, 77-84.

23 Vgl. zum Rechtswerk Minhag at-Talibin des Nawawi (gest. 676/1278) Brockelmann, Geschichte der Arabischen Literatur (GAL) G I 393 (Nr. 29), Suppl. I 680.

24 Vgl. Noor Muhammad (s. Anm. 10) 216, Contini (s. Anm. 4) 74 ff., a uch bei Scek Nur (s. Anm. 22$) 33$.

25 Zur diya vgl. Schacht, An Introduction to Islamic Law (1964) 185; auch el-Hakim, La diya et les atteintes aux personnes physiques dans le droit syrien et libanais: Annales de la faculté de droit et des sciences économiques de Beyrouth 1957, 249-287; Hamdy, Die Blutrache in Ägypten (VAR) (Diss. Wien 1967).
} 
Strafcharakter zueigen sei, habe sie nach dem somalischen Gewohnheitsrecht, das insoweit nicht vom islamischen Recht berichtigt zu werden brauchte, nur die Bedeutung eines zivilrechtlichen Schadensersatzes. Die kollektive Verantwortlichkeit des Stammesverbandes sei daher auch keine kollektive Strafe. Die Beklagten erhoben dagegen den Einwand, daß die diya gegen den ordre public (public policy) verstoße; denn das Bestreben des Staates sei darauf gerichtet, Somalia unter Aufhebung der Stammesstrukturen in einen einheitlichen $\mathrm{Na}$ tionalstaat umzuformen. Da jedoch sowohl die Verfassungsgebende Versammlung wie die Nationalversammlung die diya offensichtlich für Rechtens angesehen hatten, sah sich der Supreme Court zu keiner anderen Entscheidung in der Lage. Gegebenenfalls sei es Aufgabe der Nationalversammlung, die Verpflichtung zur Zahlung der diya gesetzlich aufzuheben, argumentierte das Gericht. Schließlich äußerte das Gericht die Ansicht, daß die diya auch im Fall einer Tötung durch einen Verkehrsunfall verlangt werden könnte, da das Gewohnheitsrecht nicht $\mathrm{zwischen} \mathrm{vorsätzlicher} \mathrm{und} \mathrm{fahrlässiger} \mathrm{Tötung} \mathrm{unterscheide,} \mathrm{sondern} \mathrm{allein} \mathrm{auf}$ die Verursachung abstelle und daher in beiden Fällen ein Anspruch auf diya entstehe. Auch sei es belanglos, ob die Tötung direkt oder durch ein Fahrzeug erfolge und ob sie in der Stadt oder auf dem Land geschehen $\operatorname{sei}^{\mathbf{2 6}}$.

3. Beispiel: In einem anderen Fall ging es um die Frage, ob ein Austritt aus einem Stammesverband möglich sei. In einer Klage auf diya, ebenfalls wegen Tötung eines Menschen bei einem Verkehrsunfall, hatte der Vertreter der beklagten Gemeinschaft beantragt, die Klage abzuweisen, da der Täter vor dem Unfall seinen Stammesverband verlassen habe. Die Vorinstanzen hatte die Möglichkeit eines Austritts aus einem Stammesverband verneint.

Nach dem Supreme Court ist die Zugehörigkeit zu einem Stammesverband rechtsgeschäftlicher Natur. Die Zugehörigkeit zu einem bestimmten Stammesverband ergäbe sich zwar mit der Geburt, bleibe aber nur aufgrund einer zustimmenden Willensäußerung des betroffenen Mitgliedes bestehen. Schon in der Vergangenheit habe nicht nur ein Stammesmitglied von dem Stammesverband ausgestoßen werden können, sondern einzelne Mitglieder hätten auch ihren Stamm verlassen können. Der vertragliche Charakter der Stammesbindungen sei durch die somalische Verfassung verstärkt worden. Die Verfassung zeige die klare Absicht, vom Stammesrecht abzurücken, ohne es ausdrücklich aufzuheben und auf den Staat nicht nur die volle Souveränität zu übertragen, sondern auch den Schutz der Rechte des einzelnen, was früher Aufgabe des Stammes gewesen sei. Nunmehr habe jedoch der Stamm seinen Charakter als ,,organische Einheit" verloren. Heute bestehe der Stamm nur noch in den Grenzen der Verfassung. Das bedeute, daß der Stamm auf dem freien Willen seiner Mitglieder beruhe. Jedes Mitglied habe daher das Recht, aus dem Stammesverband auszutreten. Die durch die Geburt begründete Vermutung der Stammeszugehörigkeit sei in allen Fällen widerlegt, in denen das Einverständnis des einzelnen, seine ,, affectio societatis“, offenkundig fehle, weil er ausdrücklich oder konkludent den Willen ausgedrückt habe, nicht mehr in der Gemeinschaft zu bleiben. Die Abwesenheit vom Stammesverband allein genügt dafür allerdings noch nicht. Eine andere Interpretation der Mitgliedschaft in einem Stamm würde Art. 26 Abs. 2 der Verfassung widersprechen, nach dem die Zugehörigkeit zu einer Vereinigung von einem entsprechenden Willen (affectio societatis) abhängt. Da sich der Täter in wirksamer Weise von seinem Stammesverband getrennt hatte, brauchte der Stammesverband keine diya zu zahlen. Die Klage wurde in diesem Fall abgewiesen ${ }^{27}$.

26 Vgl. Noor Muhammad (s. Anm. 10) 217, Scek Nur (s. Anm. 22) 34 sowie Contini (s. Anm. 4) 66; auch Contini, The Evolution (s. Anm. 22), Journal of African Law 1971, $77 \mathrm{ff}$.

27 Vgl. Noor Muhammad (s. Anm. 10) 220, Scek Nur (s. Anm. 22) 36 f., Contini (s. Anm. 4) 71 ff. sowie Contini, The Evolution (s. Anm. 22), Journal of African Law 1971, $77 \mathrm{ff}$. - Es mag allerdings mehrals zweifelhaft erscheinen, ob der historisch gewachsene Stammesverband als „Vereinigung“ (association) im Sinn des Art. 26 der Verfassung angesprochen werden kann. 
Die höchstrichterlichen Erwägungen zur diya hat der Gesetzgeber nach dem Staatsstreich von 1969 schließlich zum Anlaß genommen, die Verantwortlichkeit der Stammesgemeinschaft für die diya ganz aufzuheben und ausschließlich dem Täter allein aufzubürden. Zur Begleichung der diya darf er weder Geld sammeln noch sich Zuwendungen von Dritten machen lassen (Gesetz Nr. 67 vom 1. 11. 1970). Durch dieses Gesetz von 1970 sind die herkömmlichen Stammesstrukturen weiter geschwächt worden.

\section{Entwicklung nach dem Staatsstreich von 1969}

Auch unter dem Militärregime gehen die seit der Staatsgründung aufgenommenen Bemühungen weiter, durch Kodifizierung neuer allgemeinverbindlicher Gesetze die Rechtsordnung des Landes zu vereinheitlichen. Ein schwieriges Problem bildet dabei die Wahl der Gesetzessprache. Italienisch, Englisch und Arabisch, nicht aber das von der Mehrheit der Bevölkerung gesprochene Somali gelten als offizielle Sprachen ${ }^{28}$. Im allgemeinen erscheinen die neuen Gesetzgebungsakte im Amtsblatt auf englisch und/oder italienisch, weil für das Arabische immer noch zu wenig juristisch erfahrene Fachkräfte zur Verfügung stehen. So war das neue Strafgesetzbuch, das durch Gesetzesdekret (marsum tasrii) Nr. 5/1962 vom 16. $12.1962^{29}$ erlassen worden war und ab 2. 4. 1964 galt, erst Anfang 1973 auf arabisch im Amtsblatt veröffentlicht worden ${ }^{30}$ - zu einem Zeitpunkt also, als es bereits fast neun Jahre in Geltung war. Das Strafgesetzbuch basiert auf dem italienischen Strafgesetzbuch von $1930^{\mathbf{3 1}}$. Die neue Straf prozeßordnung wurde durch das Gesetzesdekret Nr. 1/1963 vom 1. 6. 1963 in Kraft gesetzt, galt aber erst ab 1. 8. $1965^{32}$.

\section{Das Zivilgesetzbuch von 1973}

Die bedeutendste Kodifizierung der letzten Jahre stellt das Zivilgesetzbuch dar, das unter der Bezeichnung Codice Civile Somalo auf italienisch veröffentlicht wurde und am 1. 7. 1973 in Kraft getreten ist ${ }^{33}$. Im ersten Moment könnte man glauben, daß dieses Gesetzbuch in Fortführung der bisherigen Rechtstradition den Codice Civile Italiens zum Vorbild hat. Doch mit dem Staatsstreich von 1969 war nicht nur die nationalistische Tendenz und damit die B etonung des arabisch-islamischen Elements verstärkt worden, sondern gleichzeitig war die Ideologie des arabischen Sozialismus in das politische Leben Somalias eingeführt worden. Die mehr oder weniger unmittelbare Übernahme italienischen Rechts verbot sich damit von selbst.

In der Tat erweist sich das neue Zivilgesetzbuch Somalias bei näherer Prüfung als eine weithin wörtlich gleichlautende Fassung des ägyptischen Zivilgesetzbuches von 1948/4934. So-

28 Zum Sprachproblem in Somalia vgl. Noor Muhammad (s. Anm. 10) 32 ff., Contini (s. Anm. 4) 11; auch Area Handbook for Somalia, hrsg. von Kaplan u. a. (1969) $65 \mathrm{f}$.

29 Das Gesetzesdekret Nr. 5/1962 vom 16. 12. 1962, durch welches das Strafgesetzbuch erlassen wurde, ist im Supplement Nr. 1 zum Amtsblatt Nr. 10 vom 2. 10. 1962 veröffentlicht worden.

30 Das Strafgesetzbuch wurde (auf arabisch) im Supplement Nr. 2 zum Amtsblatt (nasra rasmiya) Nr. 1/1973 vom 13. 1. 1973 veröffentlicht.

31 Zu den (italienischen) Wurzeln des StGB vgl. im einzelnen Noor Muhammad (s. Anm. 10) 307 ff., auch Contini (s. Anm. 4 ) 46 ff.

32 Vgl. zum Zeitpunkt des Inkrafttretens der StPO das Dekretgesetz Nr. 5/1965 vom 30. 3. 1965 sowie das Gesetz Nr. 10/1965 vom 1. 6. 1965. Vgl. dazu Noor Muhammad (s. Anm. 10) 308, 335 ff., Contini (s. Anm. 4) $49 \mathrm{f}$.

33 Das neue somalische ZGB von 1973 (Ǵesetz Nr. 37 vom 2. 6. 1973) ist in der italienischen Fassung des Amtsblattes, im Bolletino Ufficiale Nr. 6 vom 2. 6. 1973 veröffentlicht worden.

34 Das neue somalische ZGB hat nur 958 Artikel statt 1149 Artikel wie das ägyptische Vorbild. Das somalische ZGB ist also insgesamt 191 Artikel kürzer als das ägyptische. Bis Art. 504 hinkt das somalische ZGB nur um drei Artikel hinter dem ägyptischen ZGB zurück. 
mit liegt nun auch eine italienische Ubertragung des ägyptischen Zivilgesetzbuches vor! Wie das Beispiel des Strafgesetzbuches lehrt, ist mit einiger Verzögerung auch eine arabische Fassung des Gesetzbuches zu erwarten. Die zunächst verblüffende Ubernahme des ägyptischen ZGB bot sich um so mehr an, als es sich bei dem ägyptischen ZGB um ein erprobtes Gesetzeswerk in arabischer Sprache handelt ${ }^{35}$, das nach arabischer Ansicht nicht nur vielfältige islamische Elemente aufweist, sondern auch zahlreichen sozialistischen Rechtsideen Rechnung trägt. Im Sudan ist das ägyptische ZGB bei seiner zeitweisen Ubernahme von 1971 bis 1973 deshalb sogar ohne größere Veränderungen geradezu als das erste sozialpolitische $\mathrm{Zi}$ vilgesetzbuch im arabischen Raum gepriesen worden ${ }^{\mathbf{3 6}}$. Aber das ägyptische ZGB entsprach nicht nur den rechtspolitischen Intentionen der Militärmachthaber in Somalia, sondern erleichterte auch die Schaffung eines einheitlichen Vermögensrechtes ganz ungemein. In dem ägyptischen Zivilgesetzbuch lag eine historisch gewachsene Kodifikation vor, die in mehreren Jahrzehnten in der Praxis erprobt worden ist ${ }^{37}$. Außerdem steht mit der Úbernahme gleichzeitig eine reichhaltige Rechtsliteratur (Kommentare, Lehrbücher und Entscheidungen) zur Verfügung, wenn auch in arabisch ${ }^{\mathbf{3 8}}$.

Allerdings bedeutet die Ubernahme des ägyptischen Zivilrechts in der ehemals britischen Nordregion insofern einen scharfen Bruch mit den bisherigen Verhältnissen, als sich Somalia mit diesem Schritt eindeutig zum romanischen Rechtskreis bekennt; denn das ägyptische Zivilgesetzbuch beruht letztlich auf dem französischen Zivilrecht ${ }^{39}$. Hier liegt die Hauptschwierigkeit für eine erfolgreiche Rezeption des ägyptischen ZGB.Im Sudan hat bekanntlich dieser Bruch mit der Rechtstradition des Landes zum Scheitern des ägyptischen ZGB ganz wesentlich beigetragen ${ }^{40}$.

Es bleibt abzuwarten, ob Somalia auf Dauer Mitglied des ,,ägyptischen Rechtskreises“ bleibt. Nachdem zuletzt im Jahre 1975 Algerien das ägyptische Zivilgesetzbuch übernommen $^{41}$ und der Nordjemen 1976 das HGB von Kuwait zum Vorbild für sein Handelsgesetzbuch genommen hat, gilt heute in neun arabischen Staaten ein einheitliches Zivilrecht ${ }^{\mathbf{4}}$. Dieser Umstand ist für die Einheit der arabischen Welt von großer politischer Bedeutung. Durch die Ubernahme des ägyptischen ZGB hat das islamische Recht in Somalia eine noch stärkere Stellung erlangt. Am deutlichsten zeigt sich das Bemühen, auf die islamische Tradition als materielles Element zurückzugreifen, in der Regel, daß Lücken im Gesetz (nach Art. 1 Abs. 2 ZGB) nicht wie in Ägypten in erster Linie durch Gewohnheitsrecht auszufüllen sind, sondern wie in Syrien und Libyen durch die ,,principi del diritto islamico“, also die allgemeinen Prinzipien des islamischen Rechts und nicht durch das islamische Recht einer be-

35 Vgl. zum ägypt. ZGB etwa Linant de Bellefonds, Le droit musulman et le nouveau Code civil égyptien: Revue Algérienne 1956, I 211-222; Sanhoury, Le droit musulman comme élément de refonte du code civil égyptien: Introduction à l'étude d'un droit comparé. Recueil d'études en l'honneur d'Edouard Lambert (1938), Bd. 2, 621-640; Anderson, The Shari'a and Civil Law (The debt owed by the new civil codes of Egypt and Syria to the Shari'a): Islamic Quarterly 1954, 29-46.

36 Zeitschrift für vergleichende Rechtswissenschaft 74 (1974) 39 ff. (65) und die dort angeführten arabischen Quellen.

37 Vgl. Dilger, Das Rechtswesen in Ägypten, in: Ägypten (Erdmann-Ländermonographie), hrsg. von Schamp (1977), 509-529 (515) sowie Dilger, Das sudanesische Zivilgesetzbuch (s. Anm. 36), Zeitschrift für vergleichende Rechtswissenschaft 74 (1974) 57.

38 Vgl. etwa den Großkommentar von Sanhuri, al-wasit fi sarh al-qanun al-madani al-gadid (al-Qahira 1956-1968); auch Murqus, sarh al-qanun al-madani (al-Qahira 1967) sowie zahlreiche Lehrbücher und kommentierende Werke zu einzelnen Rechtsgebieten.

39 Vgl. dazu Dilger, Das Rechtswesen (s. Anm. 37) $511 \mathrm{f}$.

40 Vgl. Dilger, Die Stärkung des islamischen Rechts in Afrika als Folge der Emanzipation afrikanischer Mitgliedsstaaten der Arabischen Liga: Die Welt des Islams (W. I.) 1978, 153-177 (162 ff., 169), Thompson, The Failure of Continental Codes in the Democratic Republic of the Sudan, VRU $1975,407 \mathrm{ff}$.

41 Das neue algerische ZGB erging durch die Ordonnance Nr. 75-58 vom 26. 9. 1975 (Journal Officiel Nr. 78 vom 30. 9. 1975), rückwirkend in Kraft seit dem 5. 7. 1975 (Art. 1003); vgl. RabelsZ 1975, 510 sowie RabelsZ 1976, 290.

42 Ägypten (1948/49), Syrien (1949), Irak (1951), Libyen (1953), Kuwait (1961), Qatar (1971), Somalia (1973), Algerien (1975) und Nordjemen (1976). - Vgl. zu Qatar und Nordjemen Dilger, Grundbegriffe der Eigentumsordnung zwischen Wandel und Tradition auf der Arabischen Halbinsel: Zeitschrift für vergleichende Rechtswissenschaft $77(1978), 21-78(38,52)$.

43 Vgl. Art. 1 Abs. 2 ZGB in Syrien und Libyen. wo die Grundsätze des islamischen Rechts (mabadi' as-sari'a al-islamiya) im Fall von Gesetzeslücken an erster Stelle als maßgebend bezeichnet werden. vgl. dazu Dilger, Das sudanesische Zivilgesetzbuch (s. Anm. 36), Zeitschrift für vergleichende Rechtswissenschaft 74 (1974), 45 f. (auch zum Begriff „Grundsätze des islamischen Rechts“). 
stimmten Rechtsschule ${ }^{\mathbf{4 3}}$. Der Vorrang des islamischen Rechts gegenüber dem Gewohnheitsrecht ist um so bemerkenswerter, als das Gewohnheitsrecht in Somalia eine wesentlich größere Rolle spielt als in Ägypten, aber offensichtlich in Fortsetzung der vorrevolutionären Politik zurückgedrängt werden soll. Dieser Eindruck wird durch die Beobachtung verstärkt, daß das Gewohnheitsrecht im Gegensatz zu anderen arabischen Ländern ${ }^{44}$ erstaunlicherweise überhaupt nicht zur Lückenfüllung berufen wird. Vielmehr sind Gesetzeslücken nach Einschaltung des islamischen Rechts mit den Prinzipien der sozialen Gerechtigkeit (principi de giustizia sociale) ${ }^{45}$ und notfalls gemäß den Grundsätzen der equity (equita) auszufüllen ${ }^{\mathbf{4 6}}$. Die ,,Prinzipien der sozialen Gerechtigkeit“, die an die Stelle des ,,Naturrechts“ im ägyptischen ZGB getreten sind, stellen einen Terminus des arabischen Sozialismus dar und sind ein Indiz dafür, daß Somalia den Ideen des arabischen Sozialismus auch im Zivilrecht Geltung verschaffen will ${ }^{47}$.

Ferner sollen einige grundlegende Theorien aus dem islamischen Recht entlehnt worden sein. So wird in der ägyptischen Rechtsliteratur behauptet, daß die Regelung der Willkür der Rechtsausübung (vgl. Art. $5 \mathrm{ZGB})^{48}$ auf dem islamischen Recht beruhe, ebenso die sog. Lehre von den plötzlich eintretenden ,, allgemeinen außergewöhnlichen Ereignissen“ (Art. 144 Abs. 2 ZGB) ${ }^{49}$. Nach ihr kann der Richter die an sich noch mögliche Leistung aufgrund solcher unvorhergesehener Umstände, die den Schuldner so stark belasten würden, daß sie ihn mit einem drückenden, schweren Schaden bedrohen würden, nach den Umständen und nach Abwägung der Interessen beider Parteien auf ein vernünftiges Maß reduzieren. In Wirklichkeit sind beide Theorien Ausdruck modernen europäischen Rechtsdenkens und weisen einen viel zu entfernten Zusammenhang mit dem islamischen Recht auf, um aus ihm abgeleitet zu sein ${ }^{50}$. Aus dem islamischen Recht stammen dagegen die Vorschriften über die Geschäftsfähigkeit und deren Abhängigkeit vom ,,Unterscheidungsvermögen“ (Artt. 45 und $109 \mathrm{ff}$.) ${ }^{51}$, die Schuldübertragung (Artt. 312 ff.) $)^{52}$, die Schenkung (Artt. 463 ff. $)^{53}$ und das nur für Immobilien geltende und daher im Sachenrecht geregelte Vorkaufsrecht (Artt. $745 \mathrm{ff}$. $)^{54}$. Eine ty pisch scheriatrechtliche Rechtsfigur ist auch der (Ver-)Kauf und Wert während der letzten Krankheit (Artt. 474 und $475 \mathrm{ZGB})^{\mathbf{5 5}}$, der als letztwillige Verfügung ange-

44 Das Gewohnheitsrecht ('urf) wird zur Lückenfüllung z. B. in Syrien und Libyen berufen, aber auch in Ägypten und im Irak (jeweils Art. 1 Abs. 2 ZGB). - Vgl. dazu Dilger a. a. O. (s. Anm. 43).

45 Selbst im sozialistischen Sudan wurde im Fall von Gesetzeslücken nicht aufdie soziale Gerechtigkeit abgestellt; vgl. Dilger, Das sudanesische Zivilgesetzbuch (s. Anm. 36), Zeitschrift für vergleichende Rechtswissenschaft 74 (1974), $45 \mathrm{f}$.

46 Die Einbeziehung der „Billigkeit“ ('adl) geht auf das britische Recht zurück. Auch in den Vereinigten Arabischen Emiraten wird die equity zur Lückenfüllung berufen; vgl. Dilger, Grundbegriffe (s. Anm. 42), Zeitschrift für vergleichende Rechtswissenschaft 77 (1978), 32.

47 Vgl. Dilger, Eigentumsordnung und Sozialismus (s. Anm. 8), Zeitschrift für vergleichende Rechtswissenschaft 75 (1976) $179 \mathrm{f}$.

48 Vgl. Dilger, Das sudanesische Zivilgesetzbuch(s. Anm. 36), Zeitschrift für vergleichende Rechtswissenschaft 74 (1974), 59; dazu auch Anderson, The Shari’a and Civil Law (s. Anm. 35), Islamic Quarterly 1954, 33 und Sanhoury, Le droit musulman (s. Anm. 35) Bd. 2, 629.

49 Vgl. Dilger a. a. O. (s. Anm. 48).

50 Vgl. Linant de Bellefonds, Le droit musulman (s. Anm. 35), Revue Algérienne 1956, I 215 ff., der entsprechende Behauptungen zum ägyptischen ZGB überzeugend widerlegt.

51 Vgl. zur Geschäftsfähigkeit (ahlīya) und zum „Unterscheidungsvermögen“" (tamyīz) in Ägypten Artt. 44, 109; Syrien Artt. 46, 110; Irak Artt. 106, 93; Libyen Artt. 44, 109 ZGB. -Vgl. dazu Anderson, The Sharï'a and Civil Law (s. Anm. 35), Islamic Quarterly 1954, 35.

52 Vgl. zur Schuldübertragung (hawalat ad-dain) in Ägypten A rtt. 315-322; Syrien A rtt. 315-321; Irak Artt. 339-341, 349, 357 und 418; Libyen Artt. 302-309 ZGB. - Vgl. dazu Schacht, An Introduction (s. Anm. 25) 148 f.; auch Küppers, Das irakische Zivilgesetzbuch: Zeitschrift für vergleichende Rechtswissenschaft 62 (1960), II 7; Anderson, The Shari'a and Civil Law (s. Anm. 35), Islamic Quarterly 1954, 34; Sanhoury, Le droit musulman (s. Anm. 35) Bd. 2, 627, 633.

53 Vgl.zur Schenkung (hiba) in Ägypten Artt. 486 ff.; Syrien Art. 454; Irak Artt. 601 ff. ; Libyen Artt. 475 ff. ZGB. - Vgl. Schacht, An Introduction (s. Anm. 25) 157 f.; Küppers, Das irakische Zivilgesetzbuch (s. Anm. 52), Zeitschrift für vergleichende Rechtswissenschaft 62 (1960) II 13 und Anderson, The Shari'a and Civil Law (s. Anm. 35), Islamic Quarterly 1954, 42.

54 Vgl. zum Vorkaufsrecht (suf'a) in ÄgyptenArtt. 935 ff.; Irak Artt. 1128 ff.; Libyen Art. 939 ZGB (fehlt in Syrien). - Vgl. Schacht, An Introduction (s. Anm. 25) 106; Anderson, The Shari'a and Civil Law (s. Anm. 35), Islamic Quarterly 1954, 40 ff. und Sandhoury, Le droit musulman (s. Anm. 35) Bd. 2, 624.

55 Vgl. zum (Ver-)Kauf während der letzten Krankheit (bai' fi marad al-maut) in Ägypten Artt. 477, 478; Syrien Artt. 445, 446 i. V. mit Art. 877; Irak Art. 1109; Libyen Artt. 466, 467 i. V. mit Art. 920 ZGB. - Vgl. Anderson, The Shari'a and Civil Law (s. Anm. 35), Islamic Quarterly 1954, 38; Sanhoury, Le droit musulman (s. Anm. 35) Bd. 2, 625. 
sehen wird. Die Möglichkeit des Erben, die grundsätzlich über den Tod der Parteien hinaus fortdauernde Miete (Pacht) beim Tod des Mieters (Pächters) gerichtlich auflösen zu lassen (Artt. 562 ZGB) ${ }^{56}$, stellt einen Kompromiß zwischen einander entgegengesetzten islamischen Lehrmeinungen dar ${ }^{57}$.

Das Recht des Käufers, bis zum genauen Beschauen der verkauften Sache vom Vertrag zurückzutreten ${ }^{58}$, ist nach dem Vorbild der anderen modernen arabischen Zivilgesetzbücher dahingehend eingeschränkt worden, daß der Käufer zwar grundsätzlich eine genügende Kenntnis haben muß, diese aber angenommen wird, wenn der Kaufvertrag eine Bezeichnung der Kaufsache und ihrer wesentlichen Eigenschaften enthält, so daß ihre Identifizierung möglich ist (Art. 416 ZGB). Darüber hinaus bestimmt das Gesetz: Wenn in dem Kaufvertrag erwähnt ist, daß der Käufer den Kaufgegenstand kennt, bleibt das Rücktrittsrecht nur im Falle arglistiger Täuschung seitens des Verkäufers bestehen ${ }^{59}$.

Die Bestimmungen über die gemeinsame Mauer sind auf der Grenzlinie zweier Grundstücke (Art. 688 ff. ZGB) ${ }^{60}$ sind ebenfalls islamrechtlichen Ursprungs. Auch die Regelung eines Angebots unter Anwesenden in einer „Vertragsverhandlung“ (sede contrattuale) ${ }^{61}$ wird dem islamischen Recht zugeschrieben: Danach kann eine Offerte unter Anwesenden grundsätzlich nur sofort angenommen werden, ohne Zurücknahme des Angebots aber bis zum Ende der Zusammenkunft (Art. 93 ZGB). Gleiches gilt bei telefonischer oder ähnlicher Verbindung ${ }^{62}$. Nur gelegentlich fehlen typisch islamrechtliche Vorschriften, so z. B. über den hikr ${ }^{63}$ und die Pacht gegen Beteiligung an der Ernte ${ }^{64}$, besonderer Arten der Pacht, die in Somalia nicht üblich sind.

Andere Rechtsfiguren des ägyptischen Zivilgesetzbuches haben in dem somalischen Gesetzbuch nur deswegen keine Berücksichtigung gefunden, weil ihre Regelung anderen Gesetzen vorbehalten ist. So vermißt man nicht nur die Bestimmungen über die religiösen Stiftungen (waqf) ${ }^{65}$, sondern es fehlen auch das Familien- und Wohnungseigentum ${ }^{66}$, Renten ${ }^{67}$, die Úbernahme öffentlicher Dienste ${ }^{\mathbf{6 8}}$, Lebens- und Feuerversicherung ${ }^{69}$, die Teilung durch gerichtliches Verfahren ${ }^{70}$ sowie Nachlaßfragen ${ }^{71}$, die zum (noch nicht geregelten) Erbrecht gehören. Das Gesellschaftsrecht soll durch ein besonderes Gesetz geregelt werden (Art. 504 ZGB). Deshalb sind die Bestimmungen über die Gesellschaft weggelassen ${ }^{72}$. Der Arbeits-

56 Vgl. zur Auflösung von Miete (Pacht) beim Tod des Mieters (Pächters) in Ägypten Art. 601; Syrien Art. 568; Irak Art. 783; Libyen Art. 600 ZGB.

57 Vgl. Schacht, An Introduction (s. Anm. 25) 154 (hanafitisch); Sachau, Muhammedanisches Recht nach schaf ütischer Lehre (1897) 552; auch Anderson, The Shari'a and Civil Law (s. Anm. 35), Islamic Quarterly 1954, 37 und Sanhoury, Le droitmusulman (s. Anm. 35) Bd. 2, 635 f. - Vgl. außerdem in Ägypten Art. 626; Syrien Art. 593 und Libyen Art. 625 ZGB, nachdenendiePacht durch den Tod des Pächters aufgelöst wird. Anders dagegen im Irak Art. 812 ZGB.

58 Vgl. zum ,,Rücktrittsrecht der Besichtigung“ (hiy' ${ }^{\mathbf{6} a r}$ ar-ru'ya) Schacht, An Introduction (s. Anm. 25) 152. Dies gilt nach dem islamischen Recht an sich auch für die Miete, da sie als Verkauf eines Nutzens aufgefaßt wird. Doch fehlt eine entsprechende Bestimmung im ägyptischen ZGB und den von ihm abhängigen Gesetzbüchern.

59 Vgl. die gleichlautende Regelung in Ägypten in Art. 419; Syrien Art. 387; Libyen Art. 408; ähnlich im Irak Art. 517 ZGB. - Vgl. Sanhoury, Le droit musulman (s. Anm. 35) Bd. 2, 625.

60 Vgl. zur gemeinsamen Mauer in Ägypten Art. 814-818; Syrien Artt. 974, 975 (unwesentlich kürzer); Irak Artt. 1088-1091; Libyen Artt. 823-827 ZGB. - Vgl. auch Anderson, The Shar ${ }^{6} 1^{\prime}$ a and Civil Law (s. Anm. 35), Islamic Quarterly 1954, 40 und Sanhoury, Le droit musulman (s. Anm. 35) Bd. 2, 625.

61 Arab.: maglis al-'aqd.

62 Zur Annahme eines Angebotes bis zum Ende der ,,Vertragsverhandlung“ vgl. in Ägypten Art. 94; Syrien Art. 95; Irak Art. 88; Libyen Art. 94 ZGB; Kuwait Art. 113 HGB. - Vgl. auch Schacht. An Introduction(s. Anm. 25) 145; Linant de Bellefonds, Traité de droit musulman comparé I (1965) $146 \mathrm{ff}$. und Anderson, The Shari'a and Civil Law (s. Anm. 35), Islamic Quarterly $11954,35$.

$63 \mathrm{Vgl}$. Artt. 999-1014 ägypt. ZGB.

$64 \mathrm{Vgl}$. Artt. $619 \mathrm{ff}$. ägypt. ZGB.

$65 \mathrm{Vgl}$. Artt. $628 \mathrm{ff}$. und 731 ägypt. ZGB, aber auch Art. 78 somalisches ZGB.

$66 \mathrm{Vgl}$. Artt. $850 \mathrm{ff}$. ägypt. ZGB, andererseits aber Art. 718 somalisches ZGB.

$67 \mathrm{Vgl}$. Artt. $545 \mathrm{ff}$. und $741 \mathrm{ff}$. ägypt. ZGB.

$68 \mathrm{Vgl}$. Artt. $668 \mathrm{ff}$. ägypt. ZGB.

$69 \mathrm{Vgl}$. Artt. $754 \mathrm{ff}$. und Artt. $766 \mathrm{ff}$. ägypt. ZGB.

$70 \mathrm{Vgl}$. Artt. $837 \mathrm{ff}$. ägypt. ZGB.

$71 \mathrm{Vgl}$. Artt. $877 \mathrm{ff}$. ägypt. ZGB.

72 Vgl. Artt. 507 ff. ägypt. ZGB. 
vertrag fehlt ${ }^{73}$, weil das Arbeitsrecht bereits durch ein besonderes Gesetz kodifiziert worden ist (vgl. Art. 606 ZGB) dessen Erstreckung auf ganz Somalia im Jahre 1964 erfolgt ist ${ }^{74}$. Der einzige Gegenstand, der über das ägyptische Zivilgesetzbuch hinausgeht, ist die Umschreibung im Grundbuch (Artt. 938 ff. im 5. Buch des ZGB), da in Somalia ein eigenes Registerrecht noch fehlte, wie es das ägyptische Zivilgesetzbuch voraussetzt.

Abschließend bleibt darauf hinzuweisen, daß im Bereich des Vermögens- und Verfahrensrechts bei Fehlen neuer Gesetzesregelungen subsidiär noch das Recht aus der Kolonialzeit gilt, $d$. h. italienisch orientiertes Recht in der ehemals von Italien beherrschten Region sowie common law und equity bzw. indisches Recht in der früher von Großbritannien beherrschten Nordregion ${ }^{75}$.

73 Vgl. Artt. 675 ff. ägypt. ZGB.

74 Durch das Gesetz Nr. 2 vom 9. 1. 1964. - Vgl. Contini (s. Anm. 4$) 44$.

75 Vgl. Noor Muhammad (s. Anm. 10) 213 f., 287, 355, auch 209 sowie Contini (s. Anm. 4) 11, 59, 89 und 213 f. - Die Wertpapiere sind durch das Gesetzesdekret Nr. 2 vom 9. 9. 1965 gesetzlich geregelt. 


\section{Law Reform and Islam in Somalia}

\section{By KONRAD DiLger}

Since independence in 1960, when Italian and British Somaliland were united, Somalia has stressed her Islamic heritage as a means of national integration. Islamic law played, therefore, also a major role in the process of reforming and unifying the pluralist colonial legal heritage. The article shows how Islamic notions were used to modernise traditional Somali Customs (e. g. concerning blood-money, diya). The unification of private law has been achieved by the new Civil Code of 1973. The author shows this to be an almost literal reception of the Egyptian Civil Code, bringing Somalia into the "egyptian legal family" of nine Arab countries, marked by both Islamic and French civil law. Future will show whether this break with the legal tradition for the northern (formely British) part of the country will prove durable. 